\title{
Valores hematológicos y bioquímicos del axolotl (Ambystoma mexicanum) mantenidos en cautiverio en Lima, Perú
}

\author{
Haematological and biochemical values of the Mexican axolotl (Ambystoma \\ mexicanum) kept in captivity in the Lima, Peru
}

\author{
Brayan Alfonso Anaya-Lavalle ${ }^{1,2}$, Siever Morales-Cauti ${ }^{1}$
}

\section{Resumen}

El axolotl o ajolote es un anfibio caudado utilizado en numerosos estudios biomédicos por su capacidad regenerativa. Actualmente se encuentra en la categoría de peligro crítico de extinción a consecuencia de la contaminación acuática, caza indiscriminada e introducción de especies invasoras en su medioambiente natural. El objetivo del presente estudio fue determinar los rangos de parámetros hematológicos y bioquímicos de axolotls cautivos en Lima, Perú, para que puedan ser utilizados como referencia durante la evaluación clínica en laboratorios biomédicos y consultorios veterinarios de especies no convencionales. El estudio se realizó en una colección privada de axolotls en el distrito de San Juan de Miraflores, Lima. Se emplearon 27 axolotls, los cuales fueron restringidos químicamente para realizar la colecta de sangre, lográndose establecer rangos referenciales para 13 parámetros hematológicos y 10 bioquímicos.

Palabras clave: axolotl, Ambystoma mexicanum, anfibio, bioquímica, hemograma

\section{Abstract}

The axolotl is a caudate amphibian used in numerous biomedical studies for its regenerative capacity. It is currently in the category of critical danger of extinction because of aquatic pollution, indiscriminate hunting and introduction of invasive species into its

\footnotetext{
${ }^{1}$ Facultad de Ciencias Veterinaria y Biológicas, Universidad Científica del Sur, Lima, Perú

${ }^{2}$ E-mail: brnanaya@gmail.com
}

Recibido: 26 de marzo de 2021

Aceptado para publicación: 20 de octubre de 2021

Publicado: 22 de diciembre de 2021

CLos autores. Este artículo es publicado por la Rev Inv Vet Perú de la Facultad de Medicina Veterinaria, Universidad Nacional Mayor de San Marcos. Este es un artículo de acceso abierto, distribuido bajo los términos de la licencia Creative Commons Atribución 4.0 Internacional (CC BY 4.0) [https:// creativecommons.org/licenses/by/4.0/deed.es] que permite el uso, distribución y reproducción en cualquier medio, siempre que la obra original sea debidamente citada de su fuente original 
environment. The objective of the present study was to determine the ranges of haematological and biochemical parameters of captive axolotls in Lima, Perú, so that they can be used as reference values during the clinical evaluation in biomedical laboratories and veterinary clinics of unconventional species. The study was carried out in a private collection of axolotls in the district of San Juan de Miraflores, Lima. In total, 27 axolotls were used, which were chemically restricted to perform the blood collection, establishing reference ranges for 13 haematological and 10 biochemical parameters.

Key words: axolotl, Ambystoma mexicanum, amphibian, biochemistry, hemogram

\section{INTRODUCCIÓN}

El axolotl o ajolote (Ambystoma mexicanum), endémico de la ciudad de México, es un anfibio caudado de cuerpo robusto con tres pares de branquias externas. En la antigüedad fue inspiración para la creación de la leyenda del dios «Xolotl» y fuente de alimento, con propiedades medicinales para las culturas mesoamericanas (Barcat, 2018; Zapata y Solís, 2013). En la actualidad es una de especie de importancia para las investigaciones biomédicas, debido a que no experimenta metamorfosis y a que posee la capacidad de regenerar cualquier tipo de tejido (Vázquez-Molina, 2010; Zapata y Solís, 2013). Sin embargo, la especie se encuentra en la categoría de peligro crítico de extinción en México, debido a la contaminación del lago Xochimilco (único lugar donde se encuentra en estado silvestre) (Voss et al., 2015; Vázquez-Molina, 2010), la aparición de enfermedades emergentes como la chitridiomicosis (Fowler, 2012; Mendoza-Almerallaa et al., 2015), la caza indiscriminada y la introducción de especies invasoras (Zambrano, 2010). Se han desarrollado diversos programas de protección para esta especie (Voss et al., 2015; Vance, 2017).

El axolotl ha sido sujeto de múltiples investigaciones tales como, inducción de metamorfosis con hormona tiroidea (Rosenkilde et al., 1995), criopreservación de semen (Chester y Figiel., 2013), fecundación in vitro
(Mansour et al., 2011), anestesiología (Zullian et al., 2016), y creación de quimeras hematopoyéticas (Lopez, 2015), entre otras; sin embargo, los estudios hematológicos son escasos, algunos están incompletos, y otros fueron realizados con ejemplares enfermos (Wright, 2001) o con especies no emparentadas (Solis et al., 2007; Finkler, 2013).

Rosenkilde et al. (1995) proporcionaron datos cuantitativos de las líneas celulares hematológicas para axolotl y otras especies. Boutilier et al. (1992) publicaron un reporte de hematología y bioquímica sanguínea realizado en una hembra adulta de axolotl que estaba afectada por una neoplasia ovárica, siendo esta referencia el primer estudio de hematología reportado en axolotls de cautiverio. Iannacone et al. (2008) identificaron la morfología de las líneas celulares hematológicas de Aequidens mauseanus y Ambystoma mexicanum. Barriga-Vallejos et al. (2015) reportaron valores hematológicos del axolotl de Toluca (Ambystoma rivulare), en tanto que Takami y Une (2017) analizaron parámetros de bioquímica sérica y hematocrito provenientes de axolotls mantenidos en cautiverio. De todo lo anterior, se evidencia la carencia de estudios hematológicos específicos para A. mexicanum. Por tal motivo, el objetivo del presente estudio fue determinar los rangos de los valores hematológicos y bioquímicos de axolotls mantenidos en cautiverio en el distrito de San Juan de Miraflores (Lima-Perú). 


\section{Materiales y Métodos}

\section{Población y Muestra}

Previa autorización del Comité de Ética Institucional de la Universidad Científica del Sur, se obtuvieron 32 larvas de axolotls de variedad silvestre (coloración verde olivo y negro, natural de los ejemplares del lago Xochimilco), en aparente buen estado de salud, comprobado por evaluación clínica (piel brillante sin presencia de lesiones hemorrágicas, úlceras o crecimiento de hongos, branquias largas con presencia de filamentos, heces firmes en forma de frejol, sin diarrea) (Wright, 2001). Las larvas tenían 1.5 meses aproximadamente, medían $5 \mathrm{~cm}$ de largo y tenían $3 \mathrm{~g}$ de peso en promedio.

Las larvas nacieron en cautiverio en el acuario «Huancayo» del distrito de Ate, LimaPerú, el cual tenía autorización para comercializarlos en calidad de mascotas, según el reglamento nacional (Ley Forestal y de Fauna Silvestre $\mathrm{N}^{\circ}$ 2963) (SERFOR, 2015).

\section{Sitio de Estudio}

Los ejemplares fueron trasladados en bolsas plásticas llenas de agua a un casa particular del distrito de San Juan de Miraflores, Lima-Perú, donde fueron mantenidos bajo condiciones controladas durante un año, hasta que alcanzaron un peso de $50 \mathrm{~g}$ y 30 centímetros de longitud como mínimo, para poder obtener una muestra de sangre suficiente $(0.5-0.8 \mathrm{ml})$ para ser procesada en laboratorio.

Se estableció un periodo de cuarentena de 90 días para observar si alguno de los ejemplares desarrollaba síntomas de enfermedades (Cinco larvas no sobrevivieron). Cada axolotl fue alojado individualmente en recipientes plásticos de 60 por $30 \mathrm{~cm}$ y $4 \mathrm{~L}$ de capacidad, para evitar conductas de canibalismo (Wright y Whitaker, 2001d). Los recipientes fueron llenados con agua de grifo ( $\mathrm{pH} 6.5 \mathrm{y}$ densidad $1.050 \mathrm{~g} / \mathrm{ml}$ ), la cual fue tratada con solución acondicionadora para peces de acuario (Nutrafin Acua-Plus) a razón de $0.1 \mathrm{ml} / \mathrm{L}$. Se les ofreció un fotoperiodo de 12 horas de luz y oscuridad (Takami y Une, 2017). No se utilizó ningún tipo de sustrato o decoración en los recipientes, para evitar laceraciones en la piel y la presentación de timpanismo por ingesta accidental.

Se realizaron cambios de agua dos veces a la semana, retirando el $50 \%$ del volumen total de líquido y completando con agua limpia previamente acondicionada. La temperatura fue mantenida en un rango de 8.3 a $10{ }^{\circ} \mathrm{C}$ (Schad, 2008; Servin, 2011; Mena y Montes, 2014). Se realizó la limpieza de sedimento, heces y restos de muda con ayuda de un sifón para acuarios. No se utilizaron equipos de filtración automatizado debido a que las corrientes generadas por la recirculación de agua provocan lesiones y estrés en los ejemplares (Schad, 2008; Servin, 2011; Mena y Montes, 2014).

Se realizaron control de pesos y tamaño mensuales, así como análisis coprológicos por método de sedimentación (Wright y Whitaker, 2001). En dos ejemplares se encontró trofozoítos de protozoarios flagelados, los cuales no recibieron tratamiento, ya que no ocasionaron signos clínicos en los ejemplares y son atribuibles a población comensal del intestino (Wright y Whitaker, 2001).

Los axolotls fueron alimentados con presas vivas, tres veces por semana, empleando para este fin Artemia salina y Tubifex tubifex (previamente desinfectado con albendazol y metronidazol) (Browne, 2009; Servin, 2011).

\section{Colección de Muestras}

Se realizó en dos etapas para no sobrepasar el $1 \%$ de peso vivo de sangre recolectada (Wright, 2001; Campbell, 2015a) y cuando los ejemplares tenían dos años de edad. Con la primera muestra se realizó el análisis bioquímico y con la segunda, luego de tres meses, el análisis hematológico (número de 
eritrocitos, hematocrito, hemoglobina, valores absolutos y relativos de las líneas celulares de leucocitos). Los ejemplares ( 9 hembras y 18 machos) no recibieron alimento las 48 horas previas a la contención química para reducir el riesgo de emesis (Wright, 2001).

En la contención química se utilizó Eugenol (U.S.P 926) (solución a base de clavo de olor con $99.5 \%$ de pureza) diluido en 1 $\mathrm{L}$ de agua proveniente de las peceras de los axolotls (dosis: $350 \mu 1 / 1$ ) (Baitchman y Stetter, 2014). Los especímenes fueron sujetados utilizando guantes de polietileno sin talco (R\&G) y colocados en un recipiente de $20 \mathrm{x} 15 \mathrm{~cm}$ y $1 \mathrm{~L}$ de capacidad conteniendo la solución anestésica y mantenido a la misma temperatura del recinto $\left(10^{\circ} \mathrm{C}\right)$. Luego de $30-40 \mathrm{mi}-$ nutos se produjo la pérdida del reflejo de enderezamiento y se alcanzó plano quirúrgico (Baitchman y Stetter, 2014). El individuo fue posicionado ventro-dorsal en una esponja humedecida con agua del recinto y se mantuvo la humedad de la piel y branquias con agua del recinto sin anestésico (Wright et al., 2001; Baitchman y Stetter, 2014). La frecuencia cardiaca y saturación de oxígeno fue monitoreada con pulsioxímetro (EDAN H100) y Doppler fetal (Jumper JPO-1008) (Wright et al., 2001b; Baitchman y Stetter, 2014).

Se utilizó una jeringa de tuberculina de $1 \mathrm{ml}$ (25 G x 5/8"; Vanish Point), la cual se cargó con heparina de sodio (Laboratorios Rovi de $1000 \mathrm{UI} / \mathrm{ml}$ ) para impregnar las paredes, y luego se expulsó el contenido (Wright et al., 2001a; Takami y Une, 2017). La sangre ( $1 \%$ del peso vivo del animal, $0.5-0.8 \mathrm{ml})$ se tomó de las venas branquiales (Zwemer, 1991) en un minuto de tiempo cronometrado (Wright, 2001a; Campbell, 2015a) y fue almacenada en tubos de heparina de litio (Vacuette LH; Nipro) y mantenidas en refrigeración a $4{ }^{\circ} \mathrm{C}$ hasta su procesamiento (una hora después). Todos los ejemplares fueron devueltos a sus recintos luego de 30 minutos (periodo de recuperación), sin que luego de 24 horas se observen efectos secundarios y recibieron alimento.

\section{Procesamiento de Muestras}

Las muestras para bioquímica sanguínea fueron procesadas en el laboratorio privado de la Unidad de Cuidados Intensivos Veterinarios (UCIVET), ubicado en Lince, Lima, utilizando el rotor Vet Scan Avian Reptilian Profile Plus en el analizador automatizado Vet Scan Vs2 Abaxis, el cual permite analizar 10 parámetros. aspartato aminotransferasa (AST) mediante el método modificado IFCC, creatina quinasa (CK) mediante fosforilación inversa, glucosa (GLU) utilizando el método de reducción de cobre, calcio (Ca) con la reacción de Arsenazo III, fósforo (PHOS) mediante sucrosa fosforilasa, proteínas totales (TP) utilizando la reacción de Buriet, albúmina (ALB) utilizando la coloración verde de bromocresol, sodio $(\mathrm{Na})$ mediante coloración enzimática y potasio $(\mathrm{K})$ por espectrofotometría.

Las muestras para el análisis hematológico fueron remitidas al laboratorio Vet Diagnostics, Callao, Perú. Las muestras fueron procesadas manualmente empleando un microscopio óptico trinocular Eurotech de campo claro, la cámara de Neubauer y las tinciones Natt-Herrick (conteo total de leucocitos y eritrocitos) y tinción WrightGiemsa (conteo diferencial de leucocitos). Se determinó el número de eritrocitos (RBC), leucocitos (WBC), eosinófilos, basófilos, heterófilos, azurófilos y linfocitos (Campbell, 2015a; Wright, 2001). Los trombocitos no se pudieron contabilizar debido a la presencia de núcleo y la dificultad para diferenciarlos de los linfocitos (Campbell, 2015b; Wright, 2001). Para la hemoglobina (Hb) se utilizó método fotométrico de metahemoglobina azida fotométrica utilizando un hemoglobinómetro portátil Mission), el hematocrito $(\mathrm{Ht})$ se calculó mediante centrifugación de tubos microcapilares a $12000 \mathrm{rpm}$. La hemoglobina corpuscular media (HCM), concentración de hemoglobina corpuscular media (CHCM) y el volumen corpuscular medio (VCM) se calcularon mediante las fórmulas matemáticas respectivas $(\mathrm{VCM}=$ Hematocrito $(\mathrm{L} / \mathrm{L}) * 1000 /$ 
Eritrocitos*(1012/L); HCM= $\mathrm{Hb}(\mathrm{g} / \mathrm{dl}) * 10 /$ Eritrocitos * (1012/L); CHCM=Hb (g/dl) * 10/Hematocrito (L/L)).

\section{Análisis de Resultados}

Los rangos referenciales se establecieron según los lineamientos de la Sociedad Americana de Patología Clínica Veterinaria (ASVCP) para muestras de fauna silvestre, exóticos y neonatos, con tamaños muestrales mayores e iguales a 20 y menores a 40 (Kristen y Friedrich, 2012). Estos lineamientos indican que se deben descartar "valores aberrantes», puesto que son atribuidos a errores de muestreo o procesamiento. En este caso se descartaron cinco valores ( 1 de AST, 3 de glucosa y 1 de fósforo). Para el análisis de los datos se utilizó el programa informático Excel y el paquete estadístico SPSS.

\section{Resultados y Discusión}

Se determinaron rangos referen-ciales con media y desviación estándar (DE) de 13 parámetros hematológicos y 10 bioquímicos para la especie Ambystoma mexicanum (Cuadro 1). Solo existen dos reportes de parámetros hematológicos para esta especie, siendo estos los de Iannacone et al. (2008), quienes describen la morfología de las líneas celulares, y los valores relativos mas no los valores absolutos de leucocitos, y el estudio de Takami y Une (2017) quienes solo calcularon el hematocrito y la bioquímica sanguínea, de allí que es difícil comparar los resultados obtenidos. Por otro lado, Boutilier et al. (1992) presentaron valores hemáticos de una sola hembra afectada con una neoplasia ovárica.

Los valores de hematocrito $(\mathrm{Ht})$ reportados por Iannacone et al. (2008) de 8.5$33.8 \%$ son mucho más bajos que los del presente estudio (Cuadro 1), diferencias que pueden deberse a la hidratación, la dieta empleada o a efectos del anestésico utilizado. Un estudio en esturiones siberianos (Acipen- cer baerii) reveló que el Eugenol produce una pequeña elevación de los valores de hemoglobina y hematocrito (Gomulka et al., 2015). Este efecto también se observó para el hematocrito en ratas cuyos valores suboieron ante la exposición a Eugenol (Pandian et al., 2018). Por otro lado, los valores de hematocrito del presente estudio se encuentran dentro del rango de $18-47 \%$ reportado por Takami y Une (2017).

Con relación a la bioquímica sanguínea, Takami y Une (2017) reportan valores de 81$1139 \mathrm{U} / \mathrm{L}$ de AST, en tanto que en el presente estudio se encuentran en el rango de 113285 U/L. Por otro lado, los valores de glucosa de $17-55 \mathrm{mg} / \mathrm{dl}$ presentados por estos autores fueron similares a los obtenidos en este estudio (30.2-57.9 mg/dl) (Cuadro 1). Sin embargo, cabe acotar que la glucosa es un parámetro que se ve afectado por el tiempo de procesamiento de la muestra, ya que al pasar las horas las células consumen la glucosa residual obteniendo resultados inferiores. Por otro lado, los valores de creatina quinasa (CK), albumina, globulina, proteínas totales, sodio, potasio y calcio fueron similares a los presentados por Takami y Une (2017).

Por último, los valores de fósforo (1.8$3.8 \mathrm{mg} / \mathrm{dl}$, Cuadro 1) fueron inferiores a los reportados por Takami y Une (2017) de 3-6 $\mathrm{mg} / \mathrm{dl}$. Estas diferencias pueden explicarse por la producción de huevos en las hembras, ya que, en especies de aves, reptiles y anfibios, hay una tendencia a la elevación de los valores de calcio y fosforo por estimulación hormonal o formación del cascarón de los huevos (Wright, 2001a). También se ha registrado cambios en las concentraciones de fósforo y calcio en cuadros de acidosis o alcalosis respiratoria (Ghosh y Joshi, 2008). Por esta razón sería conveniente monitorear la ventilación de los anfibios anestesiados y separar parámetros de electrolitos en grupos específicos para machos y hembras, teniendo en cuenta además si el muestreo fue ejecutado en temporada de apareamiento. 
Cuadro 1. Parámetros hematológicos y bioquímicos de 27 axolotl (Ambystoma mexicanum) criados en cautiverio (Lima, Perú)

\begin{tabular}{lccc}
\hline Parámetros & Unidad & Media $\pm \mathrm{DE}$ & Rango \\
\hline Hematológicos & & & \\
Eritrocitos & $\mathrm{x} 10^{6} / \mu \mathrm{l}$ & $1.3 \pm 0.3$ & $1.0-1.6$ \\
Hemoglobina & $\mathrm{g} / \mathrm{dl}$ & $11.8 \pm 1.7$ & $10.1-15.5$ \\
Hematocrito & $\%$ & $43.5 \pm 5.7$ & $37.8-49.2$ \\
VCM & $\mathrm{fl}$ & $356.1 \pm 50.9$ & $305.3-407.0$ \\
HCM & $\mathrm{pg}$ & $97.7 \pm 21.3$ & $76.4-118.9$ \\
CHCM & $\%$ & $27.2 \pm 2.9$ & $24.3-30.0$ \\
Leucocitos & $\mathrm{x} 10^{3} / \mu 1$ & $8.8 \pm 1.0$ & $7.8-9.7$ \\
Heterófilos & $\mathrm{x} 10^{3} / \mu 1$ & $4.5 \pm 0.9$ & $3.5-5.4$ \\
Linfocitos & $\mathrm{x} 10^{3} / \mu 1$ & $3.3 \pm 1.0$ & $2.3-4.3$ \\
Monocitos & $\mathrm{x} 10^{3 / \mu 1}$ & $0.2 \pm 0.1$ & $0.05-0.3$ \\
Azurófilos & $\mathrm{x} 10^{3} / \mu 1$ & $0.3 \pm 0.2$ & $0.06-0.5$ \\
Basófilos & $\mathrm{x} 10^{3} / \mu 1$ & $0.2 \pm 0.2$ & $0.02-0.4$ \\
Eosinófilos & $\mathrm{x} 10^{3} / \mu 1$ & $0.3 \pm 0.2$ & $0.1-0.4$ \\
Bioquímica sanguínea & & & $30.2-57.9$ \\
Glucosa & $\mathrm{mg} / \mathrm{dl}$ & $44.0 \pm 13.8$ & $113.0-285.7$ \\
AST & $\mathrm{U} / \mathrm{L}$ & $199.4 \pm 86.4$ & $91.6-345.2$ \\
Creatina quinasa & $\mathrm{U} / \mathrm{L}$ & $218.4 \pm 126.8$ & $5.9-9.2$ \\
Calcio & $\mathrm{mg} / \mathrm{dl}$ & $7.6 \pm 1.7$ & $1.8-3.8$ \\
Fósforo & $\mathrm{mg} / \mathrm{dl}$ & $2.8 \pm 1.0$ & $1.7-2.1$ \\
Proteínas & $\mathrm{g} / \mathrm{dl}$ & $1.9 \pm 0.2$ & $0.5-0.8$ \\
Albúmina & $\mathrm{g} / \mathrm{dl}$ & $0.7 \pm 0.2$ & $1.1-1.4$ \\
Globulina & $\mathrm{g} / \mathrm{dl}$ & $1.2 \pm 0.1$ & $2.3-5.6$ \\
Potasio & $\mathrm{mmol} / 1$ & $3.9 \pm 1.6$ & $103.8-110.5$ \\
Sodio & $\mathrm{mmol} / 1$ & $107.1 \pm 3.4$ &
\end{tabular}

\section{Conclusión}

Los valores hematológicos y bioquímicos de los axolotls (Ambystoma mexicanum) mantenidos en cautiverio obtenidos en el presente estudio pueden utilizarse como información referencial durante evaluaciones clínicas de ejemplares criados en acuarios y colecciones privadas del Perú.

\section{Literatura Citada}

1. Baitchman E, Stetter M. 2014. Amphibians. In: West G, Heard D, Caulkett N (eds). Zoo and wild animals immobilization and anaesthesia. $2^{\text {nd }} \mathrm{ed}$. New York, USA: Jhon Willey. p 303-310.

2. Barcat JA. 2018. Bestiario biomédico: ajolote. Medicina (Buenos Aires) 78: 298-300. 
3. Barriga-Vallejos C, Hernández-Gallegos O, Hunt Von Herbing I, Lopez-Moreno $A E$, Ruiz-Gómez MD, GranadosGonzales J, Garduño-Paz MV, et al. 2015. Assessing population health of toluca axolotl Ambystoma rivulare (Taylor 1940) from Mexico using leukocyte profiles. Herpetol Conserv Bio 10:592-601.

4. Boutilier RG, Stiffler DF, Toews DP. 1992. Exchange of respiratory gases, ions, and water in amphibious and aquatic amphibians. In: Feder ME, Burggren WW (eds). Environmental physiology of amphibians. USA: University of Chicago. p 81-124.

5. Browne R. 2009. Amphibian diet and nutrition. Amphibian ark science and research. [Internet]. Available in: http:// portal.isis.org/partners/aark/researchguide/amphibian $\% 20$ husbandry/ amphibian\%20diet $\% 20 \%$ and $\%$ 20nutricion.pdf

6. Campbell TW. 2015a. Blood sample collection and preparation in amphibians. In: Campbell TW (ed). Exotic animal haematology and cytology. USA: Jhon Willey. p 181-182.

7. Campbell TW. 2015b. Peripheral blood of amphibians. In: Campbell TW (ed). Exotic animal haematology and cytology. USA: Jhon Willey. p 89-94.

8. Chester F. 2013. Cryopreservation of sperm from the axolotl Ambystoma mexicanum: implications for conservation. Herpetol Conserv Bio 8: 748-755.

9. Finkler MS. 2013. Sexual dimorphism in visceral organ mass and haematology in spotted salamanders Ambystoma maculatum before and after breeding. Copeia 13: 338-345.

10. Fowler M. 2012. Diagnosis and control of amphibian chytridiomycosis. In: Zoo and wild animal medicine. Rudolf $\mathrm{P}$ (ed). USA: Elsevier. p 217-224.

11. Ghosh AK, Joshi SR. 2008. Disorders of calcium, phosphorus and magnesium metabolism. JAssoc Physician I 56: 613-621.
12. Gomulka P, D'gowski J, W'asow T, Szczepkowski M, Czerniak E, Ziomek E, Szczerbowski A, et al. 2015. Haematological and biochemical blood profile in Russian sturgeon following propofol and eugenol anaesthesia. Turk J Fish Aquat Sc 15: 13-17. doi: 10.4194/13032712-v15 102

13. Iannacone J, Pascual G Alvariño L. 2008. Caracterización leucocitaria y niveles de hematocrito de Aequidens mauseanus (Cichlidae) y Ambystoma mexicanum (Ambystomidae): estandarización para su empleo en respuestas subletales a tóxicos. Rev Cienc 6: 9-17.

14. Kristen R, Friedrichs K. 2012. American Society of Veterinarian Clinical Pathology [ASVCP] reference interval guidelines: Determination of de novoreference intervals in veterinary species and other related topics. Vet Clin Pathol 41: 441-453.

15. Lopez D, Lin L, Monaghan JR, Cogle CR, Bova FJ, Maden M, Scott EW. 2014. Mapping hematopoiesis in a fully regenerative vertebrate: the axolotl. Blood 124: 1232-1241. doi: 10.1182/ blood-2013-09-526970

16. Mansour N, Lahnsteiner F, Patzner R. 2011. Collection of gametes from live axolotl, Ambystoma mexicanum, and standardization of in vitro fertilization. Theriotecnology 34: 354-361. doi: 10.1016/j.theriogenology.2010.09.006

17. Mena H, Montes K. 2014. Manual de procedimientos para el manejo y mantenimiento de la colonia de axolotes del laboratorio de restauración ecológica. México: Laboratorio de Restauración Ecológica del Instituto de Biología de la Universidad Autónoma de México. $34 \mathrm{p}$.

18. Mendoza-Almerallaa C, Burrowesb P, Parra-Olea G. 2015. La chiitridiomicosis en los anfibios de México. Rev Mex Biodivers 86 15: 238-248.

19. Pandian M, Padmaja R, Ravindran $R$. 2018. Effect of 4-allyl-2-methoxy-phenol (eugenol) on red blood cells in subacute restraint stress induced wistar albino rats. IOSR J Dental Med Sci 12: 81-85. 
20. Rosenkilde P, Sorenser I, Ussing AP. 1995. Amphibian haematology: metamorphosis-related changes in blood cells. Neth J Zool 45: 213-215. doi:10.1163/ 156854295X00951

21. Sarah LP, Whitaker B. 2001e. Protoza and Metazoa infecting amphibians. In: Wright KM, Whitaker BR (eds). Amphibian medicine and captive husbandry. USA: Krieger Publishing. $p$ 210-236.

22. Servin E. 2011. Manual para el mantenimiento en cautiverio y medicina veterinaria aplicada al axolotl de Xochimilco (Ambystoma mexicanum) en el zoológico de Chapultepec. Tesis de Licenciatura. México: Univ. Nacional Autónoma de México. $50 \mathrm{p}$.

23. Schad K. 2008. Amphibian population management guidelines. Amphibian ark: amphibian population management workshop [Internet]. Disponible en: http:/ /www.amphibianark.org

24. Solis ME, Bandeff JM, Yug-Wern H. 2007. Haematology and serum chemistry of Ozark and eastern hellbenders. Herpetologica 63: 285-292.

25. [SERFOR] Servicio Nacional Forestal y de Fauna Silvestre. 2015. Ley forestal y de fauna silvestre N. ${ }^{\circ} 29763$. Lima: SERFOR [Internet]. Disponible en: https://www.serfor.gob.pe/wpcontent/uploads/2016/03/1ffs-y-susreglamentos.pdf

26. Takami Y, Une Y. 2017. Blood clinical biochemistries and packed cell volumes for the Mexican axolotl (Ambystoma mexicanum). J Herpetol Med Surg 27: 104-110.

27. Vázquez-Molina A. 2010. El ajolote de Xochimilco. Ciencias 98: 54-59.

28. Vance E. 2017. The axolotl paradox. Nature 551: 286-289. doi: 10.1038/ d41586-017-05921-w
29. Voss S, Zambrano L, Woodcock M. 2015. A tale of two axolotls. BioScience 65: 1134-1140. doi: 10.1093/biosci/biv153

30. Wright KM. 2001a. Amphibian haematology. In: Wright KM, Whitaker BR (eds). Amphibian medicine and captive husbandry. USA: Krieger Publishing. p 135-144.

31. Wright KM. 2001b. Restraint techniques and euthanasia. In: Wright KM, Whitaker BR (eds). Amphibian medicine and captive husbandry. USA: Krieger Publishing. p 130-140.

32. Wright KM, Whitaker B. $2001 a$. Quarantine. In: Wright KM, Whitaker BR (eds). Amphibian medicine and captive husbandry. USA: Krieger Publishing. p 357-365.

33. Wright KM, Barnett S, Cover J. 2001b. Amphibian husbandry and housing. In: Wright KM, Whitaker BR (eds). Amphibian medicine and captive husbandry. USA: Krieger Publishing. $p$ 35-59.

34. Zambrano L, Mosig P, McKay J, Griffiths $R$, Shaffer B, Flores-Villela O. 2010. Ambystoma mexicanum. The IUCN Red List of Threatened Species. [Internet]. Available in: http://dx.doi.org/ 10.2305/iucn.uk.2010-2.rlts.t1095a3229615.en

35. Zapata MC, Solís L. 2013. Axolotl: el auténtico monstruo del Lago Xochimilco. Kuxulkab 19 41-47. doi: 10.19136/ kuxulkab.a19n36.336

36. Zullian C, Dodelet-Devillers A, Roy S, Vachon P. 2016. Evaluation of the anaesthetic effects of ms 222 in the adult Mexican axolotl (Ambystoma mexicanum). Vet Med-Czech 7: 1-7. doi: 10.2147/VMRR.S96761

37. Zwemer CF. 1991. An effective acquisition and stable containment technique for Ambystoma mexicanum whole blood. Axolotl Newsletter 20:39-40. 